\title{
Two-Dimensional Heterogeneous Dynamics at the Surface of a Colloidal Suspension
}

\author{
A. Duri, ${ }^{1, *}$ T. Autenrieth, ${ }^{1}$ L.-M. Stadler, ${ }^{1}$ O. Leupold, ${ }^{1}$ Y. Chushkin, ${ }^{2}$ G. Grübel, ${ }^{1}$ and C. Gutt ${ }^{1, \dagger}$ \\ ${ }^{1}$ HASYLAB at DESY, Notkestraße 85, 22607 Hamburg, Germany \\ ${ }^{2}$ European Synchrotron Radiation Facility, 6 rue Jules Horowitz, BP 220, 38043 Grenoble Cedex, France \\ (Received 16 September 2008; published 10 April 2009)
}

\begin{abstract}
We report on an $\mathrm{x}$-ray photon correlation spectroscopy experiment investigating the surface structure and dynamics of colloidal particles suspended in a supercooled viscous liquid. The static structure factor in the direction parallel and perpendicular to the surface reveals a more disordered structure at the surface as compared to the bulk. The particles display heterogeneous ballistic dynamics parallel to the surface. The particle dynamics in the direction perpendicular to the surface is much slower and does not show the hallmarks of ballistic motion.
\end{abstract}

DOI: 10.1103/PhysRevLett.102.145701

PACS numbers: 64.70.pv, 82.70.Dd

Heterogeneous dynamics is a prominent feature of glassy and jammed systems $[1,2]$. These systems have a disordered liquidlike state in common with very slow dynamics in which structural relaxation events occur through correlated rearrangements of particle regions, socalled cooperatively rearranging regions (CRR). The CRRs are characterized by a length scale $\xi$ which increases as the glass or jamming transition is approached [3,4], but the exact relationship between jamming and glassy state is still not identified. Because of the experimental difficulty to confirm the existence of CRRs, a confined geometry where the size of the system is comparable to $\xi$ seems to be an ideal way to get more information on this specific behavior usually observed in the bulk [5], and the free surface is such a confined geometry. Moreover, it is important to identify whether or not the breaking of symmetry and the interactions at the interface play an important role in ultraslow dynamics as has been proposed, e.g., for the surface glass transition [5-7]. Colloidal particles embedded in a highly viscous liquid are a good model system for studying heterogeneous dynamics as ballistic motion, and a peculiar compressed exponential form of the intermediate scattering function has been reported before for bulk colloidal systems [8] and nanoparticles in polymer melts [9].

The samples consist of silica particles dispersed in polypropylene glycol $\left(T_{g} \approx 186 \mathrm{~K}\right)$ with an average molecular weight of $4000 \mathrm{~g} / 1$ (PPG 4000 purchased from Aldrich). By measuring capillary wave relaxations we determined the viscosity of the colloidal suspension at $T=250 \mathrm{~K}$ to be $100 \mathrm{~Pa}$ s, which is in good agreement with values found for pure bulk PPG [10]. The silica particles are prepared by polycondensation of tetraethoxy-silane according to a Stöber synthesis [11] and surface stabilized in a second reaction step with 3-trimethoxy-silyl-propyl-methacrylate. We expect the interaction to be close to a hard sphere system. The samples have been characterized by means of small angle $x$-ray scattering experiments. A diluted suspension (volume fraction $\phi \approx 1 \%$ ) was used to deduce the particle radius $(R=270 \pm 32 \AA)$, and taking a con- centrated sample (volume fraction $\phi \approx 25 \%$ ), the interparticle correlations in the bulk (interparticle mean distance $d \approx 660 \AA$ ) were measured. The slow dynamics in our system is caused by the high viscosity of the supercooled solvent.

To investigate the structure and the dynamics of the concentrated suspension at $T=250 \mathrm{~K}$ at the surface, we performed $\mathrm{x}$-ray photon correlation spectroscopy experiments under grazing incidence conditions using the coherent x-ray beam of the ID10A beam line at the European Synchrotron Radiation Facility with a photon wavelength of $\lambda=1.55 \AA$ [see Fig. 1(a)]. The energy bandpass of the $\mathrm{Si}(111)$ monochromator leads to a longitudinal coherence length $\xi_{l}=\lambda^{2} / \Delta \lambda \approx 1 \mu \mathrm{m}$. A pinhole with a diameter of $20 \mu \mathrm{m}$ is placed upstream of the sample in order to obtain a collimated and transversely partially coherent beam. The beam is then tilted vertically by a mirror onto the sample surface resulting in an incident angle $\alpha_{i}=0.12^{\circ}$, which is below the critical angle of total external reflection. This leads to an evanescent wave with a penetration depth $l$ of about $100 \AA$ providing surface sensitivity on subsingle particle level, since $R>l$ holds. The colloidal suspension has been studied in an apparatus consisting of two nested chambers. The outer cell was evacuated for thermal isolation and the inner one contained a stainless steel trough of $120 \mathrm{~mm}$ diameter and $0.2 \mathrm{~mm}$ height. Series of typically 500-1000 speckle images have been recorded at a regular time interval of about $2.7 \mathrm{~s}$ (exposure time plus read out) by a charge-coupled device $(\mathrm{CCD})$ camera with $1340 \times 1300$ pixels and a pixel size of $20 \times 20 \mu \mathrm{m}^{2}$.

We deduced the static structure factor from the time average of the speckle pattern. Because the capillary waves contribute to the static intensity, we introduce a static surface form factor $F_{S}^{2}\left(q_{r}, q_{z}^{t}\right)$, which depends on both the wave vector transfer parallel to the surface, $q_{r}$, and the wave vector transfer perpendicular to the surface, $q_{z}^{t}$, within the liquid, taking the refractive index $n$ of the material into account [12]. By using the distorted wave Born approximation we write for the form factor 
(a)
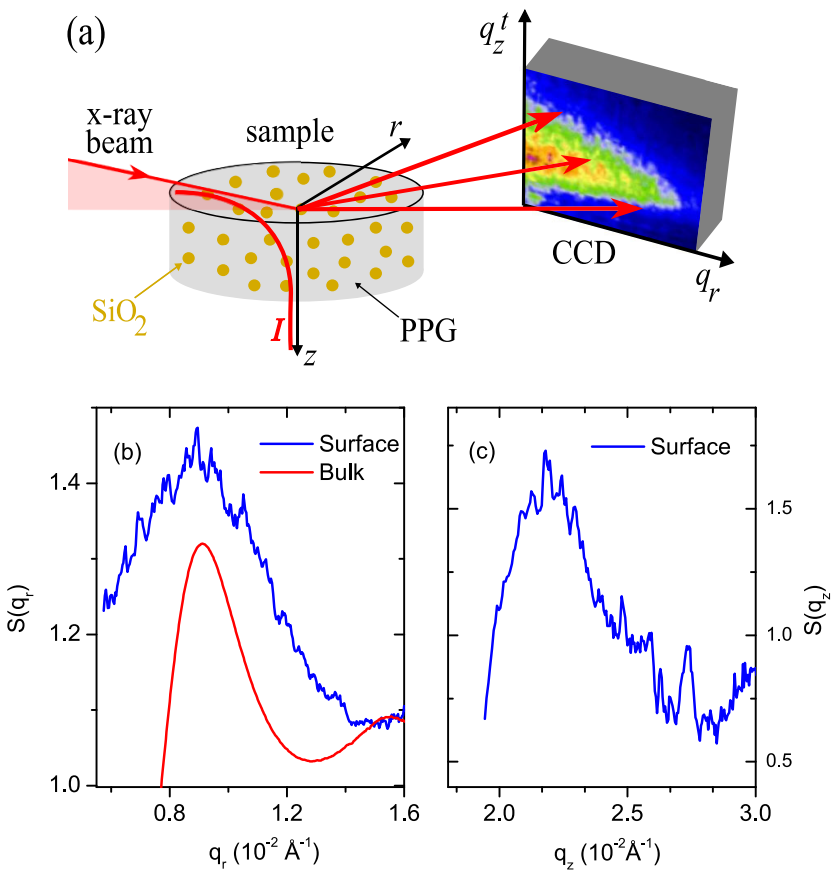

FIG. 1 (color online). (a) Schematic setup of the experiment. (b) Surface structure factor $S$ as a function of the wave vector transfer parallel to the surface. The lower curve is the bulk structure factor. (c) Surface structure factor $S$ as a function of the wave vector transfer perpendicular to the surface, $q_{z}$. The curves in (b) and (c) are scaled such that $S=1$ for the largest $q_{r}$ and $q_{z}$ values (not shown).

$$
\begin{aligned}
F_{S}^{2}\left(q_{r}, q_{z}^{t}\right) \propto & \frac{T\left(\alpha_{i}\right) T\left(\alpha_{f}\right)}{\left|q_{z}^{t}\right|^{2}}\left[\left|k_{0}^{2}\left(1-n^{2}\right)\right|^{2} \frac{k_{B} T}{\gamma q_{r}^{2}}\right. \\
& \left.+\Delta \rho_{2}^{2} V_{\text {part }}^{2} N \tilde{F}^{2}\left(q_{r}, q_{z}^{t}\right)\right],
\end{aligned}
$$

where the first term describes the scattering from capillary waves and the second term contains the particle form factor $\tilde{F}^{2}\left(q_{r}, q_{z}^{t}\right)$ in surface geometry. $\tilde{F}^{2}\left(q_{r}, q_{z}^{t}\right)$ has been numerically evaluated by taking also the absorption effect of the evanescent wave in the liquid into account. $T_{i}\left(\alpha_{i}\right)$ and $T_{f}\left(\alpha_{f}\right)$ are the transmission functions determined by the incident $\left(\alpha_{i}\right)$ and the scattering $\left(\alpha_{f}\right)$ angles, $k_{0}$ is the modulus of the incident wave vector, $k_{B}$ is the Boltzmann constant, $T$ is the temperature of the sample, $\gamma$ is the surface tension of the liquid, $\Delta \rho_{2}$ is the difference in electron densities between a silica particle and the surrounding liquid, $V_{P}$ is the volume of one particle, and $N$ is the number of particles per unit area. Averaging $F_{S}^{2}\left(q_{r}, q_{z}^{t}\right)$ over $q_{z}^{t}$ and $q_{r}$ and dividing each of the obtained results by the measured intensity yields the two distinct surface structure factors, $S\left(q_{r}\right)$ and $S\left(q_{z}\right)$, respectively, presented in Figs. 1(b) and 1(c) - the last one being plotted as a function of $q_{z}$ assuming $n=1$. Although the contribution of background scattering did not allow for a proper normalization of the structure factors, we can still discuss the shape properties of the obtained curves. The surface structure factor $S\left(q_{r}\right)$ and the bulk structure factor peak at the same position in reciprocal space $q_{r} \sim 9.5 \times$ $10^{-3} \AA^{-1}$, indicating a similar mean interparticle distance in the surface plane and in the bulk of about $2 \pi / q_{r} \approx$ $660 \AA$. However, the corresponding width of the surface and bulk structure factors are strikingly different. The surface structure factor has a width of approximately $4.4 \times$ $10^{-3} \AA^{-1}$, which is almost 3 times larger than the width of the bulk structure factor. This shows that the distribution of correlation lengths at the surface is broader; i.e., the liquid structure at the surface is more disordered than the one in the bulk.

The structure factor perpendicular to the liquid surface $S\left(q_{z}\right)$ [Fig. 1(c)] also exhibits a peak reflecting a liquidlike correlation between the position of the surface and the mean position of the first layer of particles in the liquid. $S\left(q_{z}\right)$ peaks at $q_{z} \sim 2.2 \times 10^{-2} \AA^{-1}$, which corresponds to a length scale of about $290 \AA$, a value close to the mean particle radius of $270 \AA$. Interestingly, the structure factor displays nearly the same width $\left(\sim 3 \times 10^{-3} \AA^{-1}\right)$ as the one parallel to the surface confirming the pronounced disordered state. These results demonstrate that the topmost layer of the concentrated silica suspension exhibits a liquidlike structure with the lack of one dimension leading to a more loose-packed particle arrangement than in the bulk.

Having characterized the static structure, we now analyze the dynamic properties of the particles at the surface. We applied the time-resolved correlation method [13] under grazing incidence conditions that allowed us to obtain the temporal resolution of the surface dynamics. The instantaneous degree of intensity correlation between pairs of speckle images, $c_{I}$, as a function of both the time delay $\tau$ between images and the absolute time $t$ has been calculated according to

$$
c_{I}\left(q_{r}, q_{z}^{t}, t, \tau\right)=\frac{\left\langle I_{p}(t) I_{p}(t+\tau\rangle_{p \in q_{r}, q_{z}^{t}}\right.}{\left\langle I_{p}(t)\right\rangle_{p \in q_{r}, q_{z}^{t}} I_{p}(t+\tau\rangle_{p \in q_{r}, q_{z}^{t}}}-1,
$$

where $I_{p}(t)$ is the scattered intensity at pixel $p$ and time $t$. The brackets denote the average over rectangular regions of pixels corresponding to a mean value of $q_{r}$ and $q_{z}^{t}$. The sample age $t=0$ is defined by the first image recorded by the CCD camera. The degree of correlation is displayed in Fig. 2(a) at $T=250 \mathrm{~K}$ for several $\tau$ values and for $q_{r}=$ $6.3 \times 10^{-3} \AA^{-1}$ and $q_{z}=2.1 \times 10^{-2} \AA^{-1}$.

The $c_{I}$ curves exhibit random small drops superimposed by a larger bump (around $t=420 \mathrm{~s}$ ), indicating the predominance of sudden changes in the speckle patterns and suggesting therefore the presence of intermittent rearrangement events [14] at the sample surface. The temporal fluctuations of the dynamics can be quantified by calculating the variance of $c_{I}, \sigma_{c_{I}}^{2}=\overline{\left(c_{I}-\overline{c_{I}}\right)^{2}}$ in analogy to the dynamical susceptibility $\chi_{4}$, which is often studied in theoretical and numerical works on glass forming and 

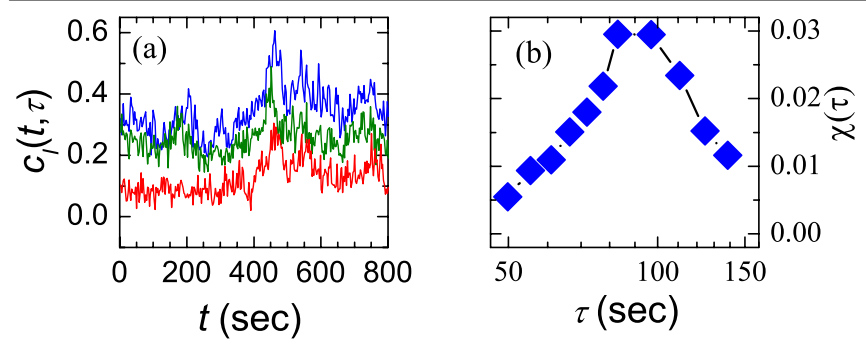

FIG. 2 (color online). (a) Temporal fluctuations of the instantaneous degree of intensity correlation, $c_{I}$, for $q_{r}=$ $6.3 \times 10^{-3} \AA^{-1}, q_{z}=2.1 \times 10^{-2} \AA^{-1}$. From the top to the bottom, $\tau=2.8,28$, and 83 s. (b) Normalized noise-free variance $\chi$ as a function of $\tau$ and for the same $q_{r}, q_{z}$ as in (a).

jammed systems $[3,15] . \sigma_{c_{l}}^{2}(\tau)$ has been corrected by subtracting the noise contribution due to the finite number of pixels over which $c_{I}$ is calculated [16] and normalized by the squared amplitude of the intensity autocorrelation function $g_{2}-1$ [17]. The function $\chi(\tau)$ is calculated between $0 \leq t \leq 800 \mathrm{~s}$ and plotted in Fig. 2(b) as a function of $\tau$ and for the same $q_{r}$ and $q_{z}$ values as for the $c_{I}$ curves shown in Fig. 2(a). $\chi(\tau)$ exhibits a pronounced peak proving the existence of dynamical heterogeneities in a confined configuration and supporting therefore the picture of the existence of cooperative rearranging regions as observed for many glassy or jammed systems $[3,4,14-18]$.

Following [19] we can assume that $N \propto N_{\text {scatt }} \times \chi_{\text {max }}$, where $N$ is the number of particles moving cooperatively in a single CRR and $N_{\text {scatt }}$ is the number of particles in the scattering volume. The rather high value of $\chi_{\max } \sim 3 \times$ $10^{-2}$ indicates that there are approximately 30 CRRs present at the illuminated surface with each CRR containing approximately $9 \times 10^{5}$ particles. We note that the here observed value of $\chi_{\max }$ is similar to bulk measurements of a colloidal gel [18], where also a small number of large CRRs was observed.

We calculated the intensity autocorrelation function $g_{2}\left(q_{r}, q_{z}, \tau\right)-1$ which characterizes the average dynamics, by performing the temporal average of $c_{I}$. Figure 3 shows $g_{2}(\tau)-1$ at $T=250 \mathrm{~K}$ for fixed $q_{z}\left(\sim 10^{-2} \AA^{-1}\right)$ and for several $q_{r}\left(6.5,8.0\right.$, and $\left.9.4 \times 10^{-3} \AA^{-1}\right)$.

We found that the functions are well described by a compressed exponential (represented by a line in Fig. 3), $\exp \left[-\left(\tau \Gamma_{r}\right)^{\beta}\right]$, where $\beta \approx 2$ is the compression exponent and $\Gamma_{r}$ is the relaxation rate in the $q_{r}$ direction. From Fig. 4(a) we see that $\Gamma_{r}$ is proportional to $q_{r}$, indicating a ballistic motion of the particles parallel to the surface, i.e., a linear growth of the average particle displacement with time. The value of the compression exponent is quite unexpected because it is commonly equal to 1.5 $[8,17,20]$. However, an exponent of 2 can be obtained by using a model with a Gaussian distribution of particle displacements in which a typical particle displacement after one event has to be smaller than the length scale probed $1 / q_{r}[8,17]$.

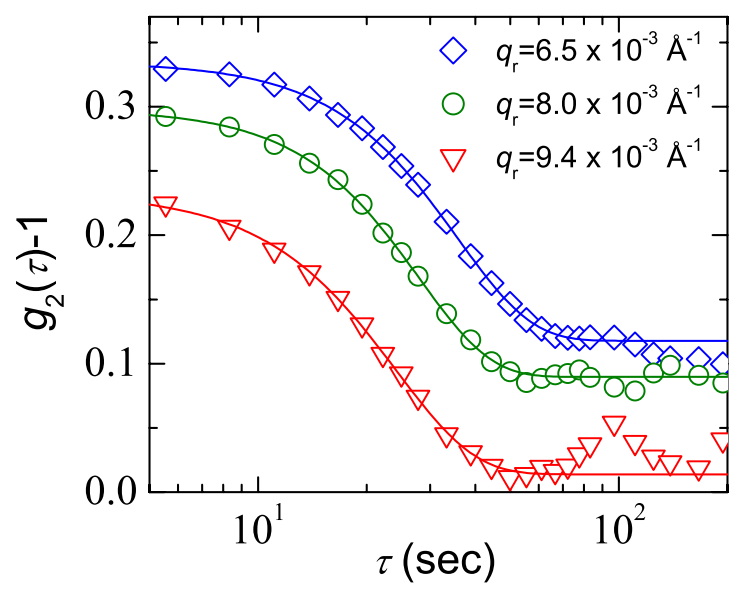

FIG. 3 (color online). Intensity autocorrelation function $g_{2}(\tau)-1$ for a fixed $q_{z}\left(\sim 10^{-2} \AA^{-1}\right)$ and for three different $q_{r}$ values. The lines are fits with a compressed exponential decay.

We can compare the measured relaxation rate with the relaxation rate of a freely diffusing particle given by $\Gamma_{0}=$ $D_{0} q_{r}^{2}$. The free diffusion coefficient is calculated using the measured viscosity and particle radius and we obtain $D_{0}=$ $6.8 \times 10^{-17} \mathrm{~m}^{2} / \mathrm{s}$. Figure 4(b) shows the ratio $\Gamma_{0} / \Gamma_{r}$. The ballisticlike dynamics of the particles is 1 order of magnitude slower than the dynamics of a freely diffusing particle. Stress relaxation and elasticity are commonly believed to be responsible for dynamical heterogeneities and ballistic type of motions. Models using local deformation fields induced by stress sources have been developed for explaining the dynamic features observed [21,22]. Recent surface sensitive $\mathrm{x}$-ray scattering experiments revealed that the solvent PPG 4000, but also other glass forming liquids, displays pronounced low-frequency elastic behavior at the surface in the supercooled phase [23-25]. The elasticity becomes visible as a transition in the surface dynamics from capillary modes to elastic modes at time scales of seconds and longer. For PPG 4000 a low-frequency elastic

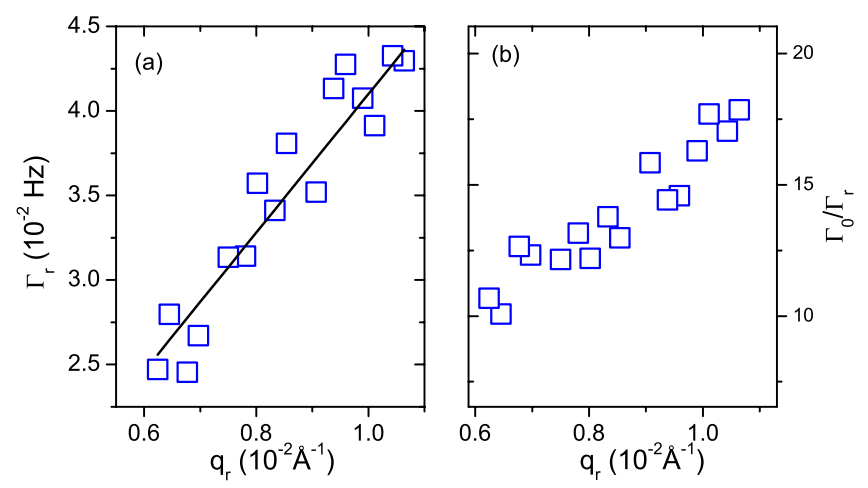

FIG. 4 (color online). (a) Relaxation rate $\Gamma_{r}$ in the $q_{r}$ direction, i.e., parallel to the surface. The solid line is a fit with $\Gamma_{r} \propto q_{r}$. (b) Relaxation rate $\Gamma_{0}$ calculated for a freely diffusing particle normalized to the measured relaxation rate $\Gamma_{r}$ in the $q_{r}$ direction. 

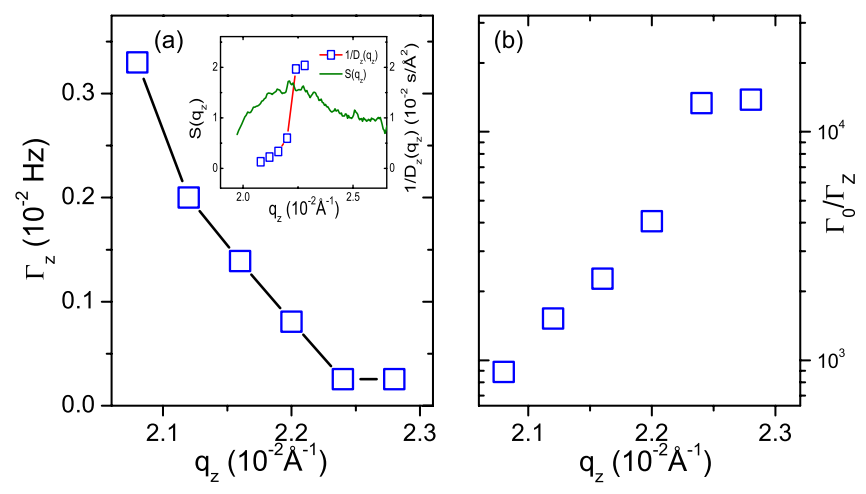

FIG. 5 (color online). (a) Relaxation rate $\Gamma_{z}$ in the $q_{z}$ direction, i.e., perpendicular to the surface. The solid line is a guide for the eyes only. Inset: The structure factor $S\left(q_{z}\right)$ in $z$ direction and the inverse of the $q$-dependent diffusion coefficient $D_{z}\left(q_{z}\right)$ calculated from the data in (a). (b) Relaxation rate $\Gamma_{0}$ calculated for a freely diffusing particle normalized to the measured relaxation rate $\Gamma_{z}$ in the $q_{z}$ direction.

modulus of $500 \mathrm{~Pa}$ has been reported at $T=250 \mathrm{~K}$ [23]. This elasticity provides a mechanism for the coupling of the solvent to the colloidal particles which is responsible for the dynamics observed. That is, the particles at the surface are moving in a viscoelastic medium and the motion of the particles induces local stress fields which can be supported due to the elastic properties of the solvent.

The relaxation rates $\Gamma_{z}$ in the $q_{z}$ direction perpendicular to the surface have been deduced from the intensity autocorrelation functions calculated for a fixed $q_{r}$ and several $q_{z}$ vectors (data not displayed). Assuming that the motions in both directions are independent, we write for the overall relaxation rate $\Gamma=\Gamma_{r}+\Gamma_{z}$. With this and the observed $q$ behavior, we can quantify both relaxation times. The behavior of $\Gamma_{z}$ shows a very different $q$ dependence by comparison with $\Gamma_{r} . \Gamma_{z}$, plotted in Fig. 5(a), is now inversely proportional to $q_{z}$ around the structure factor peak $\left(q_{z} \sim 2.2 \times 10^{-2} \AA^{-1}\right)$. The inset of Fig. 5(a) shows the structure factor $S\left(q_{z}\right)$ in the $z$ direction and the inverse of the $q$-dependent diffusion coefficient $D_{z}\left(q_{z}\right)=D_{0} q_{z}^{2} / \Gamma_{z}$ calculated from the data. We find that $1 / D_{z}\left(q_{z}\right)$ is only weakly reminiscent of the static structure factor $S\left(q_{z}\right)$.

Figure 5(b) displays the ratio $\Gamma_{0} / \Gamma_{z}$. We note that the dynamics perpendicular to the surface is 3-4 orders of magnitude slower than the free bulk diffusion which reflects the strong interaction between the free surface and the particles in the first layer. The results show that the particle motions parallel and perpendicular to the free surface are very different regarding both the type of motion and the relaxation times. This implies that the free surface induces a decoupling of the particle dynamics parallel to the surface from the particle dynamics underneath in the bulk suspension.

In conclusion, x-ray photon correlation spectroscopy under grazing incidence was used to determine the structure and the dynamics of the topmost layer of colloidal particles suspended in a highly viscous liquid. The structural data show a more disordered state at the surface than in the bulk. Two different types of dynamics have been identified. Parallel to the surface, the particle motion is ballistic and the dynamics is heterogeneous, supporting the existence of cooperative rearranging regions. The heterogeneity is attributed to the viscoelastic properties of the solvent. The dynamics perpendicular to the surface is much slower due to strong surface-particle interactions, and the $q$ dependence is different from the direction parallel to the surface.

*Present address: IATE, UMR 1208, INRA-CIRAD-UMIISupagro, 2 place Pierre Viala, 34060 Montpellier Cedex 01, France.

†christian.gutt@desy.de

[1] M. D. Ediger, J. Phys. Chem. 100, 13200 (1996).

[2] Soft and Fragile Matter, Nonequilibrium Dynamics, Metastability and Flow, edited by M. E. Cates and M. R. Evans (IOP, Bristol, 2000).

[3] N. Lacevic et al., J. Chem. Phys. 119, 7372 (2003).

[4] L. D. C. Jaubert et al., J. Stat. Mech. (2007) P05001.

[5] J. S. Sharp and J. A. Forrest, Phys. Rev. Lett. 91, 235701 (2003).

[6] C. J. Ellison and J. M. Torkelson, Nature Mater. 2, 695 (2003).

[7] Z. Fakhraai and J. A. Forrest, Phys. Rev. Lett. 95, 025701 (2005).

[8] C. Caronna et al., Phys. Rev. Lett. 100, 055702 (2008).

[9] H. Guo et al., Phys. Rev. Lett. 102, 075702 (2009).

[10] J. Cochrane et al., Polymer 21, 837 (1980).

[11] W. Stöber et al., J. Colloid Interface Sci. 26, 62 (1968).

[12] S. K. Sinha et al., Phys. Rev. B 38, 2297 (1988).

[13] L. Cipelletti et al., J. Phys. Condens. Matter 15, S257 (2003).

[14] E. Pitard, Phys. Rev. E 71, 041504 (2005).

[15] D. Chandler et al., Phys. Rev. E 74, 051501 (2006).

[16] A. Duri et al., Phys. Rev. E 72, 051401 (2005).

[17] A. Duri and L. Cipelletti, Europhys. Lett. 76, 972 (2006).

[18] V. Trappe et al., Phys. Rev. E 76, 051404 (2007).

[19] C. Toninelli et al., Phys. Rev. E 71, 041505 (2005).

[20] S. Streit et al., Phys. Rev. Lett. 98, 047801 (2007).

[21] L. Cipelletti et al., Faraday Discuss. 123, 237 (2003).

[22] J.-P. Bouchaud and E. Pitard, Eur. Phys. J. E 6, 231 (2001).

[23] Y. Chushkin et al., Europhys. Lett. 83, 36001 (2008).

[24] Z. Jiang et al., Phys. Rev. Lett. 98, 227801 (2007).

[25] M. Sikorski, Ph.D. thesis, University of Hamburg, 2008, http://www-library.desy.de/cgi-bin/showprep. pl?desy-thesis-08-013. 